Cahiers de recherches médiévales

\title{
La « jeunesse » de Jeanne d'Arc dans les traités en
}

\section{sa faveur}

\section{Françoise Michaud-Fréjaville}

\section{(2) OpenEdition}

Édition électronique

URL : https://journals.openedition.org/crm/730

DOI : $10.4000 / \mathrm{crm} .730$

ISSN : 1955-2424

Éditeur

Honoré Champion

\section{Édition imprimée}

Date de publication : 1 juin 2005

Pagination : 171-177

ISSN : $1272-9752$

\section{Référence électronique}

Françoise Michaud-Fréjaville, "La « jeunesse » de Jeanne d'Arc dans les traités en sa faveur », Cahiers de recherches médiévales [En ligne], 12 spécial | 2005, mis en ligne le 28 juin 2008, consulté le 15 décembre 2022. URL : http://journals.openedition.org/crm/730 ; DOI : https://doi.org/10.4000/crm 730 


\title{
酸M
}

\author{
$-14-$ \\ La «jeunesse » de Jeanne d'Arc dans les traités en sa faveur ${ }^{1}$
}

À dix-sept ans, elle avait chassé les Anglais d'Orléans et mené le roi Charles VII jusqu'à Reims ; à dix-neuf ans, elle tint tête aux juges de Rouen et alla dignement à la mort : c'était une toute jeune fille que Jeanne d'Arc. Des témoins des séances du tribunal de Rouen s'attachèrent, lors du procès en annulation, à souligner la contradiction entre la finesse, la prudence (c'est-à-dire la sagesse) des réponses de l'accusée et son âge réel. Maître André Marguerie souligna : «elle était jeune [...] quoique prudente en ses réponses $»^{2}$ et Pierre Cusquel rappela que «malgré ses presque vingt ans, elle était toute simple, et [...] ignorante du droit, bien qu'elle répondît avec prudence $»^{3}$. Dans une étude précédente au sujet de la désignation anthroponymique de Jeanne ${ }^{4}$, j'avais déjà souligné le flou entretenu, me paraissait-il, entre la mise en avant de la jeunesse de l'héroïne et l'usage du sobriquet La Pucelle, la jeune fille, aussi lorsque m'est parvenue l'invitation à participer à ces journées consacrées à Jeanne d'Arc, mon sujet m'est apparu évident : essayer d'analyser l'argument de la jeunesse dans le système de mise en pièce de la condamnation de Rouen, à travers les traités qui furent utilisés par les promoteurs du procès « en nullité ».

Les sources sont donc la série des traités publiée par Duparc dans son second volume du Procès en nullité, à laquelle j'ai ajouté le De adventu Johanne de Jacques Gélu dont l'édition et la traduction sont un des chantiers du Centre Jeanne d'Arc d'Orléans et certains des traités édités par Lanery d'Arc au siècle dernier.

Les mentions relevées chez les divers auteurs m'ont amenée à privilégier trois aspects mis en avant par les théologiens (aspects qui n'ont rien de théologiques) lorsqu'ils ont usé de l'argument de lajeunesse en utilisant les termes d'adulescens, juvencula, juvenis puella, paupercula juvenis. D'abord la jeunesse juridique, ensuite la fragilité physique personnelle auxquelles l'argument général du sexe faible a pu se trouver joint, enfin la simplicité de l'ignorance primitive de l'être non encore complètement formé. Il faudra bien conclure sur l'usage terriblement ambigu dans ces traités du terme puella, avec ou sans majuscule.

\footnotetext{
${ }^{1}$ Les références aux traités et au procès en nullité sont celles de l'édition de P. Duparc, Procès en nullité de la condamnation de Jeanne d'Arc, Paris Klincksieck 1977-1988 (Société de l'Histoire de France), abrégée ici : Procès en nullité. D'autres traités, non republiés par Duparc, ont été conservés, en particulier ceux de Maître Theodore de Lelier, Paul Pontanus, Jean l'Ermite et Guy de Verseilles, édités par Lanery D'Arc, Mémoires et consultations en faveur de Jeanne d'Arc par les juges des procès en réhabilitation, Paris 1889. Ici : Lanery. Le traité de Jacques Gélu est cité d'après Arch. dép. Isère, B 3139.

${ }^{2}$ Procès en nullité, t. I, p. 454.

${ }^{3}$ Ibid., p. 452.

${ }^{3}$ Procès en nullité, t. I, p. 454.

${ }^{3}$ Ibid., p. 452.

${ }^{4} \mathrm{~F}$. Michaud-Fréjaville, «Dans son pays on l'appellait Jeannette ... », dans Genèse médiévale de l'anthroponymie moderne, Discours sur le nom: normes, usages, imaginaires $\left(V I^{e}-X V I^{e}\right.$ siècles), t. IV, Tours 1997, p. 163-177, réimpr. supra, article ${ }^{\circ} 12$.
}

Cahiers de Recherches Médiévales (XII -XV s.), 12spé, 2005 
Le thème de la minorité peut être rapidement évacué, comme le firent d'ailleurs les demandeurs dans la production des motifs de droit. Ils exposèrent que la procédure des juges de Rouen n'était pas valable, entre autres raisons parce que ces derniers n'avaient pas pris en considération «la minorité de cette fille qui n'aurait pas dû sans curateur être forcée de parler en justice», dicte filie etatem minorem, que sine curatore judicio sisti cogi non valuit ${ }^{5}$. Seul Thomas Basin a fait un sort assez particulier à cet argument mais son propos n'est pas toujours lumineux. $\mathrm{Si}$, à propos de l'appel possible au Pape refusé par les juges de Rouen, il laisse filer cette expression: Autem Puella ut dixi minor annis et juris ignara ${ }^{6}$, on peut se demander s'il s'agit réellement de minorité légale ou de jeunesse inexperimentée, en revanche, plus loin il traite - et lui seul donc - des délicates questions de majorité relative et totale, et bien sûr à ce moment le doute sur les termes n'est plus permis. Basin fait appel aux subtilités du droit romain sur la majorité à 25 ans $^{7}$ : dans des causes civiles et non criminelles, les mineurs(es) de moins de 25 ans (ou de 24 ans dans certains cas) doivent avoir des curateurs et procureurs plus aptes que les accusés à saisir les nuances de l'argumentation et surtout, par définition, moins prompts à l'emportement car ils ne sont pas sujets à la juvenila calor. Cependant, pour les «bénéfices et autres matières spirituelles », les majeurs de 14 ans sont légitimés à agir et poursuivre en tribunal. Qu'en est-il de la cause de Jeanne, qui est de «foi et spirituelle»? Hoc enim non obstat, nam certe cum ibi loquatur de beneficialibus et spiritualibus causis, per hoc patet textum illum intellegi debere non de criminalibus sed de civilibus tantum 8 . L'argumention n'est réellement pas très forte et Basin n'insiste guère, il lâche prise après avoir simplement souligné qu'il était toujours possible que pour une personne de moins de 25 ans la peine fût atténuée, voire fît l'objet d'une rémission. Ce qui a posteriori est une réflexion lénifiante plutôt cruelle pour Jeanne.

En vérité l'argumentation juridique de l'absence de curateurs, qui a été également utilisée par Jean l'Ermite dans sa réfutation point par point des articles du procès de Rouen', ne tient pas car à 19 ans on est majeur au Moyen Âge (depuis 12 ans pour les filles), à moins de faire valoir que pour les femmes tout acte et toute action doivent être autorisés par le père ou le mari, ce qui dans le cas de Jeanne d'Arc était aventuré : le père de Jeanne était bien loin du tribunal de Rouen. On ne saurait dire que ce passage du traité de Basin soit vraiment le meilleur.

À son tour, la jeunesse fragile aggravée par la féminité, était-elle vraiment un bon argument pour remettre en question le procès de Rouen?

On sait que Jacques Gélu, qui écrit dans l'été de 1429 le De adventu Johanne, en ignorant bien sûr ce qui allait advenir d'elle par la suite, a justifié, parmi d'autres explications, l'apparition de la jeune fille comme volonté de Dieu de montrer sa puissance. «Dieu choisit les faibles (infirmes au sens propre) pour confondre les

${ }^{5}$ Procès en nullité, t. II, p. 11.

${ }^{6}$ Procès en nullité, t. II, p. 168.

${ }^{7}$ Ibid., t. II, p. 177-180.

${ }^{8}$ Ibid., t. II, p. 179.

${ }^{9}$ Lanery, p. 78 , réponse de Jean Lermite au dixième argument du procès de condamnation : defensor intervenire potest maxime, aut in casu prcesenti intervenire debuit consultor, seu director ipsius Johannce. 
forts $»^{10}$. Comme il ne convient pas à une femme, surtout une enfant ou une jeune fille, à cause de la fragilité et de la pudeur de son sexe d'être chef d'une armée, Il a particulièrement élu Jeanne afin de fustiger l'orgueil des Anglais: «Dieu, pour confondre et humilier leur orgueil qui s'étendait jusqu'au ciel, s'est opposé à eux per juvenculam [...] omni fragilitati subjectam ${ }^{11}$. C'est pourquoi il n'a pas envoyé un ange, il Lui «suffisait de prendre le moyen le plus faible et le plus chétif, pour confondre les moyens les plus forts des hommes $»^{12}$. On pense que le traité de Gélu date de juin 1429, l'auteur ne fait donc que constater les toutes premières victoires royales! Par la suite, la "fragilité » de Jeanne après les épisodes de la reprise des villes de la Loire, la chevauchée du sacre, les blessures, la prison et la mort ne pouvait plus guère être utilisée comme argument de sensiblilité opposant une pauvre et tendre victime à d'horribles juges. Les propos des autres défenseurs de Jeanne s'infléchirent donc.

Gerson se contente pour marquer l'opposition du physique et de l'âge entre le roi, ses capitaines, ses hommes d'armes et Jeanne - que tous s'inquiètent de devoir suivre - du diminutif muliercula, « un petit bout de femme» en quelque sorte. Que se passerait-il si ces capitaines chevronnés étaient vaincus sous son commandement? Quod evenire poterat si sub una muliercula militantes vici fuissent per hostes proca$\operatorname{cissimos}^{13}$ ? Gerson a choisi un terme qui ne l'engage en aucune façon dans un développement sur la faiblesse de la jeunesse féminine, surtout adolescente.

Cette dernière fut cependant abordée par certains de nos théologiens. Peu usèrent de véritable sensiblerie en plaignant la victime aux fers : quod puella debilis compedibus ferreis detineretur ${ }^{14}$. Mais de cette évidente délicate jeunesse, ils affirmèrent la successive présence normale puis la disparition miraculeuse. L'œuvre de Dieu a transcendé chez la fillette les lois de l'humaine nature non pas au moment historique de la mission publique mais bien auparavant. Jean de Montigny a relevé très nettement le parallélisme entre l'annonciation mariale et la soumission de Jeanne à ses voix : Cum interrogaretur quare sibi plus quam alteri facte sunt revelationes, respondit "quod sic placuit Domine». Quod autem esset parvula, constat quia puelle tredecimorum annorum, ut dicit, cum primum apparitiones sibi facte fuerunt $^{15}$. S'étant soumise aux ordres de ses voix dès ses 13 ans, quelques années plus tard, au moment de son épopée, la petite Jeanne n'est plus réductible aux catégories ordinaires. Jean Bochard faisant le même rapprochement avec l'âge de la vierge Marie (Maria puella) y inclut tout naturellement une citation du Magnificat: «il a élevé les humbles et abaissé les puissants $»^{16}$.

Hélie de Bourdeilles ${ }^{17}$, en suivant un raisonnement analogue, rejoint une partie des réflexions de l'archevêque d'Embrun, peut-être récupère-t-il les termes même de Gélu, en insistant sur l'infériorité féminine : ab etate et fragili sexu ipsius puelle

${ }^{10}$ Jacques Gélu, De adventu Johanne, Arch. dép. Isère, B 3139, fol. 5. Les traductions sont d'Olivier Bouzy (Centre Jeanne d'Arc, Orléans).

${ }^{11} I$ bid., fol. 11 : «par le moyen d'une jeune fille soumise à une totale faiblesse. »

${ }^{12}$ Ibid., fol. 14.

${ }^{13}$ Procès en nullité, t. II, p. 36.

${ }^{14}$ Lanery, p. 20 : argument de Théodore de Lelier (1452).

${ }^{15}$ Procès en nullité, t. II, p. 273.

${ }^{16}$ Ibid., t. II, p. 261

${ }^{17}$ Ibid., t. II, p. 61 et suiv. 
unde mulier inferior est viro et plus loin, en citant Chrysostome, [...] et sic fragilior viro et est sexus muliebris incautus et mollis [...] et debilis. Si les femmes ne sont pas les égales des hommes dans les travaux de la maturité, qu'en peut-il être de ceux de l'âge tendre ? Mais c'est pour faire valoir que si Jeanne était à l'époque des premières « voix » in tali fragili sexu constituta, elle devint par sa confiance en la parole de Dieu totalement autre. Ce théologien - à la différence de Montigny - se garde néanmoins de toute comparaison directe avec Marie et s'en tient strictement à l'éclosion du papillon johannique en qui la fragilité s'est transformée en virtus guerrière: viguerit et tam ardua et bellicosa opera, confisa de Domini virtute, agere presumpserit qualia viri illustrissimi... Cette mutation véritablement complète, physique mais aussi morale et intellectuelle, s'opéra pour Jeanne dans bien d'autres domaines et nous y reviendrons, mais il faut remarquer qu'elle s'inscrit dans la droite tradition de l'épitre de Paul aux Hébreux: la foi qui entraîne les créatures humaines leur donne tous les courages (He, XI, 32-40).

Finalement nous le voyons, sur les dix-huit textes examinés quatre ont traité de la fragilité de la jeunesse et les trois qui sont postérieurs à l'épopée ont détourné le sujet pour faire ressortir davantage le caractère unique de l'histoire de Jeanne, sans doute irréductible à un modèle à suivre. Cette particularité est avant tout un signe de la puissance de Dieu. Ce signe que les juges de Rouen par aveuglement haineux et partisan, par orgueil de puissants abaissés ont négligé. Auraient-ils pu être mieux alertés par la sagesse des réponses de Jeanne si contradictoires avec l'immaturité, le propre de la jeunesse?

Que Jeanne soit à la fois «simple et jeune » pour Jean d'Aulon ${ }^{18}$, «jeune simple et innocente » selon Thibaut de Termes ${ }^{19}$, «ignorante du droit» ainsi que le dit Basin et comme en a témoigné également Nicolas de Houppeville à Rouen en $1452^{20}$, est manifeste. Les traités usent de termes identiques: Martin Berryuer évoque une simplicem puellam ${ }^{21}$ et une simplex et juvenis puellam, pene idiota dans une formule fort ambiguë où inculture, pathologie et sexisme sont difficilement discernables les uns des autres ${ }^{22}$. Or cette même Jeanne si simple se révèle tout à la fois capable de réponses mesurées, réfléchies ${ }^{23}$, de réparties qui font mouche, de subtilités juridiques inattendues comme la réfutation des juges de Rouen et l'appel au Pape. Cette illiterata a placé ses défenseurs, une fois de plus, dans une situation délicate: comment utiliser en la faveur de Jeanne une jeunesse sans calcul, sans culture, intacte des compromissions. Pire encore comment répondre aux accusations qui relevaient en elle des défauts comme cette présomption «ce vice habituellement inhérent à la jeunesse $»^{24}$ qui figurait en seconde place dans les articles de la condamnation de la Pucelle?

${ }^{18} I$ bid., t. I, p. 473

${ }^{19}$ Ibid., t. I, p. 404. Thibaut d'Armagnac, sire de Termes.

${ }^{20} I b i d$., t. I, p. 144 : « elle était simple, ignorante du droit et n'était pas en état de se défendre dans ce procès $»$ (trad., t. IV p. 124).

${ }^{21}$ Ibid., t. II, p. 336.

${ }^{22}$ Ibid., t. II, p. 223

${ }^{23}$ Selon Paul Pontanus : Cum esset juveni mulier, tenbatur explicite articulos fidei sure et asserere (Lanery, p. 55.)

${ }^{24}$ Élie de Bourdeilles citant saint Bernard, Procès en nullité, t. II, p. 117. 
L'obscurité de la naissance de Jeanne et son manque d'éducation que la maturité ne pouvait avoir encore compensés sont tour à tour relevés par J. Gélu, Élie de Bourdeilles Jean Bochard et Guillaume Bouillé dans des termes très proches, dont l'origine est peut être commune mais encore difficile à cerner. Jacques Gélu parle de celle que Dieu trouva bon d'envoyer au secours du roi : «une adolescente élevée et nourrie derrière son troupeau (adulescentulam puellam de post festante nutritam et ereptam), non éduquée à l'école des maîtres, non plus que par les conversations des prud'hommes ou par l'enseignement des docteurs $»^{25}$. Le traité de l'archevêque d'Embrun a-t-il été l'inspirateur de Bourdeilles quand celui-ci rappelle la modestie extrême de la naissance de la Pucelle et reprend le thème de la bergère sans éducation? On peut le penser: ex infimis parentibus orta, inter rudes educata, et post pecora custodienda in campis conversata et a nullo hominum edocta vel inducta ${ }^{26}$.

Le traité de Gélu fut-il également connu de Jean Bochard? Cela paraît plus douteux. Ce dernier, au cours du même raisonnement dont j'ai parlé plus haut où il est question de l'élévation des humbles, évoque Marie et Jeanne (moins abruptement que je le fais ici cependant), en contrepoint de la chute des superbes Anglais : simplicissima tridecim circiter annorum virgine puelle, in campum absque multa hominum frequentia, inter oves et pecora multa, atque de post festantes a Deo vocata ${ }^{27}$. La Jeanne bergère était déjà un thème répandu et n'entre pas dans le propos de cette présente communication, en revanche il me semble important de souligner la constance mise ici à faire des seules pécores, à la conversation bruyante mais limitée, les interlocutrices des heures de solitude de la supposée pastourelle.

Finalement, dans ce même ordre d'idée, Guillaume Bouillé assimile la rusticité des bêtes à celle des soldats, aussi peu éducatrices l'une que l'autre pour une paupercula juvenis, in prima etate nutrita inter bubulcos et postea inter armigeros ${ }^{28}$. Jeanne pouvait bien s'exclamer comme Jérémie ${ }^{29}: A ! A ! A !$ Domine ecce nescio loqui, quia puer ego sum! Les théologiens complétaient aisément les versets: et dixit Dominus ad me "nolis dicere puer sum" / quoniam ad omnia quae mittam te ibis / et universa quaecumque mandavero tibi loqueris ( "mais le seigneur répondit " ne dit pas je suis une enfant" mais va vers tous ceux à qui je t'enverrai, et tout ce que je t'ordonnerai dis-le» $(J r$. I, 6-7). Le caractère prophétique de la mission de Jeanne, si on le reconnaissait évidemment, rendait inutile toute discussion sur sa jeunesse, son ignorance et sa présomption: Dieu parlait par sa bouche. Il n'y avait pas à aller au-delà. Le Seigneur seul pouvait avoir inspiré les fortes déclarations sur l'appel au Pape qui firent l'admiration de Thomas Basin ${ }^{30}$.

Mais l'ignorance et le manque de maturité pouvaient par ailleurs expliquer certaines incohérences des réponses et de la conduite de Jeanne au cours du procès. Ils permettaient en particulier d'aborder l'affaire fort embarrassante de la cédule

${ }^{25}$ J. Gélu, op. cit., fol. 5 : Placuit [...] regi succurere per adulescentulam puellam de post festante nutritam et ereptam, non scola magistrali, non conversacione prudentium, non instructione doctorum informatam.

${ }^{26}$ Procès en nullité., II, p. 61.

${ }^{27} I$ Ibid., t. II, p. 261

${ }^{28}$ Ibid., t. II, p. 321.

${ }^{29} I d$. : Excusabat enim se quod erat simplex puella, non apta hujusmodi opus ad quo mittebatur sicut legitur de Jeremia clamante : A ! A ! A, etc.

${ }^{30}$ Ibid., t. II, p. 167-168. 
d'abjuration, et l'argument fut longuement utilisé par Thomas Basin ${ }^{31}$ : Opportet igitur quod ille qui judicandus est relapsus per ante heresim abjuraverit, quod quis facere non censetur nisi intelligat quod abjurat. L'évêque de Lisieux en arrive à utiliser cinq fois en vingt lignes le verbe (non) intellegere. Mais il est évident que la possibilité pour Jeanne de se tromper ne peut être rapprochée de l'argument que j'ai cité précédemment, c'est à dire celui du caractère prophétique de la parole johannique, à moins de reconnaître que Dieu l'avait abandonnée et donc condamnée. On sait que Basin trouva une solution fort peu satisfaisante dont l'exposé déborderait du sujet $^{32}$. En voulant rationaliser par l'excuse d'une jeunesse ignorante les contradictions de la fin du procès de Rouen, on risquait d'anéantir la construction qui plaçait le projet de Dieu à l'origine et au cour de l'action de la Pucelle ${ }^{33}$. Il valait mieux ne pas insister sur cet aspect de la personne de celle qui devenait déjà une martyre.

Élie de Bourdeilles qui s'était engagé sur le terrain de l'âge tendre a trouvé des formules assez remarquables pour en sortir. Il faut ici compléter les balancements entre la Jeanne d'avant les voix et celle d'après : à l'ignorance et la solitude succédèrent la réflexion et la parole persuasive auprès du roi, à la faiblesse du sexe inférieur, la force et la victoire militaire, le berger David devint le roi David ${ }^{34}$. Il n'a pas pu cependant se tirer à son tour avec succès de l'affaire de l'abjuration: Jeanne n'ayant que 19 ans ne disposait pas de la totalité de la force d'âme et de cœur et elle a eu peur. L'argument est humain et non prophétique, Dieu reste muet. Un des mystères de Jeanne et de ses contradictions ne peut être percé.

On n'en sort pas : traiter directement de la jeunesse, faire en quelque sorte vibrer la corde sensible de l'innocence martyrisée était incompatible avec la stature supérieure de l'héroïne qu'attestaient à la fois ses actions et la qualité de la plupart de ses réponses à ses juges, y compris la rétractation héroïque qui la conduisit tout droit au bûcher. En outre Jeanne n'était pas en son temps le seul exemple de maturité précoce consacrée à Dieu et rapidement retournée à la maison du Père : Louis de Toulouse (23 ans ) et Pierre de Luxembourg (18 ans) étaient morts - eux déjà en odeur de sainteté, sexe et naissance obligent - à des âges comparables au sien. Mais en vérité, d'un côté sa jeunesse ne se prêtait que difficilement à un discours efficace contre les juges de Rouen alors que, de l'autre, les suppliques de la famille d'Arc, cristallisées autour de la figure pathétique d'Isabelle [Rommée], la rejetaient vers l'enfance, vers la jeune génération. C'est pourquoi je me demande si en utilisant uniquement dans leurs traités le terme de Puella, Jean Bochard et Jean de Montigny n'ont pas usé d'un stratagème tout à la fois signifiant et subtil, pour provoquer avec discrétion la commisération due envers la martyre («Nous avons brûlé une sainte ») sans s'engluer dans l'impossible distinction entre la faiblesse de l'âge tendre et une force divine à éclipse.

${ }^{31}$ Ibid., t. II, p. 180-181

${ }^{32}$ Thomas Basin, Histoire de Charles VII., éd. Ch. Samaran, Paris 1964 (1933) (Les classiques de l'histoire de France au Moyen Âge), t. I, p. 163. Voir: B. Guenée, Entre l'Église et l'Etat, quatre vies de prélats français à la fin du Moyen Âge, Paris 1987, p. 301-435.

${ }^{33} \mathrm{~F}$. Michaud-Fréjaville, "Autour du bûcher de Jeanne», dans Cahiers de Recherches Médiévales (XII $-X V^{e}$ siècles), $\mathrm{n}^{\circ} 3,1997, \mathrm{p} .135$, réimpr. infra, article $\mathrm{n}^{\circ} 18$.

${ }^{34}$ Procès en nullité, t. II, p. 97. 
Puella chez eux ne serait pas alors le sobriquet «La Pucelle», doté dans nos éditions modernes d'une majuscule en latin et traduit du terme archaïsant en français, mais uniquement le substantif « fille, jeune fille», destiné à imprégner le lecteur de la jeunesse de l'héroïne sans en revendiquer très ouvertement un caractère onomastique personnel. En effet, l'anthroponyme La Pucelle est utilisé sous cette forme française médiévale dans le texte original latin d'un certain nombre de traités et dans les procès, conjointement avec l'usage de puella, sans contexte de nom propre. Tout me semble à revoir. Au terme de cette assez peu satisfaisante enquête sur le vocabulaire de la jeunesse dans les traités s'ouvre un ample et nouveau chantier de relecture des textes du $\mathrm{XV}^{\mathrm{e}}$ siècle et de leur utilisation dans l'historiographie déjà riche de l'héroïne française. 